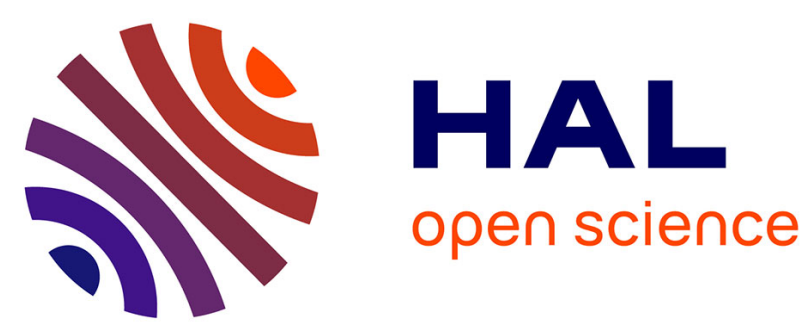

\title{
Increasing the dilution rate can globally stabilize two-step biological systems
}

Jérôme Harmand, Alain Rapaport, Denis Dochain

\section{To cite this version:}

Jérôme Harmand, Alain Rapaport, Denis Dochain. Increasing the dilution rate can globally stabilize two-step biological systems. Journal of Process Control, 2020, 95, pp.67 - 74 . 10.1016/j.jprocont.2020.08.009 . hal-02926040v2

\section{HAL Id: hal-02926040 \\ https://hal.inrae.fr/hal-02926040v2}

Submitted on 1 Oct 2020

HAL is a multi-disciplinary open access archive for the deposit and dissemination of scientific research documents, whether they are published or not. The documents may come from teaching and research institutions in France or abroad, or from public or private research centers.
L'archive ouverte pluridisciplinaire HAL, est destinée au dépôt et à la diffusion de documents scientifiques de niveau recherche, publiés ou non, émanant des établissements d'enseignement et de recherche français ou étrangers, des laboratoires publics ou privés.

\section{(ㅇ)(1) $\$$}

Distributed under a Creative Commons Attribution - NonCommercial - NoDerivatives $\mid 4.0$ 


\title{
Increasing removal rate can globally stabilize two-step biological systems
}

\author{
J. Harmand ${ }^{\mathrm{a}, *}$ A. Rapaport ${ }^{\mathrm{b}}$ D. Dochain ${ }^{\mathrm{c}}$ \\ a INRAE, Univ Montpellier, LBE, 102 avenue des Etangs, 11100, Narbonne, \\ France. \\ ${ }^{\mathrm{b}}$ INRAE, Univ. Montpellier, MISTEA, Montpellier SupAgro, France. \\ ' ICTEAM, Univ. Cath. Louvain, Belgium.
}

\begin{abstract}
We revisit two-step mass-balance models of biological processes as met to describe numerous biological systems including the anaerobic digestion or the nitrification process in view of its global stabilization. We show that when a bi-stability occurs, it can be possible to globally stabilize the dynamics toward an unique positive equilibrium by increasing the removal rate. We give sufficient conditions on the growth functions of the model for this situation to appear. This illustrates that for biological multi-step reactional systems, increasing the residence time (e.g. decreasing the input flow rate) may not be the only way to stabilize the dynamics.
\end{abstract}

Key words: Anaerobic digestion, nitrification, chemostat, multi-stability, stabilization.

\section{Introduction}

In most of continuous cultures, it is well known that increasing the removal rate (or equivalently reducing the residence time inside the reactors) can destabilize the dynamics, in the sense that it enlarges the attraction basin of the wash-out equilibrium. This can be easily shown on the classical mathematical model of the chemostat, whatever the kinetics includes inhibition or not (see [6]). For growth inhibited by the substrate, bi-stability systematically occurs

\footnotetext{
* Corresponding author.

Email addresses: jerome.harmand@inrae.fr (J. Harmand), alain.rapaport@inrae.fr (A. Rapaport), denis.dochain@uclouvain.be (D. Dochain).
} 
for large values of the input concentration of substrate. This feature has practical impacts on positive equilibrium (when it exists) because it cannot be globally stable, and the dynamics can conduct the system to the wash-out of the biomass, when the state belongs to the attraction basin of the washout equilibrium. Ways to guarantee a global stability is either to fix a lower removal rate, which is penalizing for the performance of the process, or to control the removal rate with a feedback loop, which temporarily diminishes the removal rate when the state is far from the positive steady state $[1,8,9,16]$. In any case, the removal has to be reduced at a certain stage.

Here we consider a more complex reaction scheme that are two-step systems, as met for instance in many models representing the anaerobic digestion process [4] or the nitrification process [19]. For these systems we show that there exist situations presenting a bi-stability for which increasing (and not decreasing) the removal rate conducts the system to a globally asymptotically stable steady state, in opposition to classical stabilizing practices.

In the paper, we denote by $\mathbb{R}_{+}$the set of non-negative numbers and by $\mathbb{R}_{+, \star}$ the set of positive numbers.

Let us consider the general mathematical model of a two-step mass-balance biological process, given by the following equations:

$$
\left\{\begin{array}{l}
\dot{x}_{1}=\mu_{1}\left(s_{1}\right) x_{1}-\alpha D x_{1} \\
\dot{s}_{1}=-\mu_{1}\left(s_{1}\right) x_{1}+D\left(s_{1}^{i n}-s_{1}\right) \\
\dot{x}_{2}=\mu_{2}\left(s_{2}\right) x_{2}-\alpha D x_{2} \\
\dot{s}_{2}=-\mu_{2}\left(s_{2}\right) x_{2}+\mu_{1}\left(s_{1}\right) x_{1}+D\left(s_{2}^{i n}-s_{2}\right)
\end{array}\right.
$$

where the parameter $D$ denotes the removal rate.

This model is presented under the original form proposed in [4]. The first reaction involves a microbial species of concentration $x_{1}$ which grows on a substrate of concentration $s_{1}$ with a monotonic specific rate $\mu_{1}$. The incoming flow fed the culture with substrate of concentration $s_{1}^{i n}$. The second reaction involves a second microbial species of concentration $x_{2}$ which grows on another substrate of concentration $s_{2}$, with a specific growth rate denotes $\mu_{2}$. This reaction is also fed by the first one which produces the second substrate. In addition, the incoming flow rate may contain (or not) substrate of concentration $s_{2}^{\text {in }}$. The parameter $\alpha \in(0,1)$ reflects the fact that the effective removal rate of the biomass is impacted by a retention inside the tank, differently to the abiotic resource. Here, the yield coefficients of the transformations of substrate $s_{i}$ into biomass $x_{i}(i=1,2)$, and of the production of substrate for the second reaction by the first one, have been all kept equal to 1 (this is always possible without any loss of generality, by a right choice of the concentration units). 
In many biological systems, such as the anaerobic digestion or the nitrification processes, it is often reported in the literature that the second reaction is inhibited by large values of $s_{2}$, which amounts to consider the following hypotheses.

Assumption 1 The functions $\mu_{1}$, $\mu_{2}$ belong to $C^{1}\left(\mathbb{R}_{+}, \mathbb{R}_{+}\right)$and fulfill the following properties.

(i) $\mu_{1}$ is increasing on $\mathbb{R}_{+}$with $\mu_{1}(0)=0$.

(i) There exists $\hat{s}_{2}>$ such that $\mu_{2}$ is increasing on $\left[0, \hat{s}_{2}\right)$ and decreasing on $\left(\hat{s}_{2},+\infty\right)$, with $\mu_{2}(0)=0$ and $\mu_{2}(+\infty)=0$.

The model (1) has a cascade structure: the first reaction is independent of the second one and the $\left(x_{1}, s_{1}\right)$ sub-system follows the classical (mono-specific) chemostat model. However, the $\left(x_{2}, s_{2}\right)$ sub-system is more complex to study as it receives substrate from the first reaction and $\mu_{2}$ is non-monotonic. This model and some of its variants has been already well studied in the literature $[3,4,13]$, depending on the operating parameters $\left(s_{1}^{i n}, s_{2}^{i n}, D\right)$. In particular, it has been shown that the dynamics may exhibit a multiple-stability, and the complete operating diagram has been established in $[14,19]$. The purpose of the present work is to complement those studies, investigating how to adapt the value of the removal rate $D$ to ensure a global stability of the dynamics. For sake of completeness, we first recall in the next section the set of possible asymptotic behaviors of the model.

\section{Stability analysis}

Let us first denote, for convenience,

$$
\left.\left.\mu_{1}^{m}:=\sup \left\{\mu_{1}\left(s_{1}\right) ; s_{1} \in \mathbb{R}_{+}\right)\right\}, \mu_{2}^{m}:=\sup \left\{\mu_{2}\left(s_{2}\right) ; s_{2} \in \mathbb{R}_{+}\right)\right\}=\mu_{2}\left(\hat{s}_{2}\right)
$$

(where $\mu_{1}^{m}$ could be equal to $+\infty$ ).

We define the break-even concentration $\lambda_{1}$ associated to the first reaction as the function

$$
\lambda_{1}(D):=\mu_{1}^{-1}(\alpha D), \quad \alpha D<\mu_{1}^{m} .
$$

Then, we define the following quantity

$$
\overline{s_{2}^{i n}}(D):=s_{2}^{i n}+ \begin{cases}s_{1}^{i n}-\lambda_{1}(D), & \alpha D<\mu_{1}\left(s_{1}^{i n}\right) \\ 0, & \alpha D \geq \mu_{1}\left(s_{1}^{i n}\right)\end{cases}
$$


that is playing an important role in the analysis of the equilibria, as an "effective" input concentration for the second reaction.

We define also the break-even concentrations $\lambda_{2}^{-}, \lambda_{2}^{+}$associated to the second reaction as functions such that

$$
\left[\lambda_{2}^{-}(D), \lambda_{2}^{+}(D)\right]:=\left\{s \in \mathbb{R}_{+} ; \mu_{2}(s) \geq \alpha D\right\}, \quad \alpha D \leq \mu_{2}^{m} .
$$

One has the following result about equilibria of system (1) and their stability.

Proposition 1 The asymptotic behavior of the solutions of system (1) with initial condition in $\left(\mathbb{R}_{+*} \times \mathbb{R}_{+}\right)^{2}$ is given by one of the following cases.

(1) When $\alpha D>\max \left(\mu_{1}\left(s_{1}^{\text {in }}\right), \mu_{2}^{m}\right)$, any solution converges to the "double wash-out" steady-state $E^{0,0}:=\left(0, s_{1}^{\text {in }}, 0, s_{1}^{\text {in }}\right)$.

(2) When $\max \left(\mu_{1}\left(s_{1}^{\text {in }}\right), \mu_{2}\left(s_{2}^{i n}\right)\right) \leq \alpha D \leq \mu_{2}^{m}$, the solution converges either to $E^{0,0}$ or to the equilibrium $E^{0, \star}:=\left(0, s_{1}^{i n},\left(s_{2}^{i n}-\lambda_{2}^{-}(D)\right) / \alpha, \lambda_{2}^{-}(D)\right)$, except for initial conditions on a set of null measure

(3) When $\mu_{1}\left(s_{1}^{i n}\right)<\alpha D \leq \mu_{2}^{m}$ and $s_{2}^{\text {in }} \leq \lambda_{2}^{+}(D)$, any solution converges to $E^{0, \star}$.

(4) When $\mu_{1}\left(s_{1}^{\text {in }}\right)>\alpha D>\mu_{2}\left(s_{2}\right)$ for any $s_{2}<\overline{s_{2}^{\text {in }}}(D)$, any solution converges to $E^{\star, 0}:=\left(\left(s_{1}^{\text {in }}-\lambda_{1}(D)\right) / \alpha, \lambda_{1}(D), 0, \overline{s_{2}^{i n}}(D)\right)$.

(5) When $\alpha D<\mu_{1}\left(s_{1}^{\text {in }}\right), \alpha D \leq \mu_{2}^{m}$ and $\left.\overline{s_{2}^{i n}}(D)\right)>\lambda_{2}^{+}(D)$, the solution converges either to $E^{\star, 0}$ or to the positive equilibrium $E^{\star, \star}:=\left(\left(s_{1}^{i n}-\right.\right.$ $\left.\left.\lambda_{1}(D)\right) / \alpha, \lambda_{1}(D),\left(\overline{s_{2}^{i n}}(D)-\lambda_{2}^{-}(D)\right) / \alpha, \lambda_{2}^{-}(D)\right)$, except for initial conditions on a set of null measure.

(6) When $\alpha D<\mu_{1}\left(s_{1}^{i n}\right), \alpha D \leq \mu_{2}^{m}$ and $\lambda_{2}^{-}(D)<\overline{s_{2}^{i n}}(D) \leq \lambda_{2}^{+}(D)$, any solution converges to $E^{\star, \star}$.

This result has already been proved in $[3,4,13]$, and we recall quickly here the arguments of the proof based on the study of the single chemostat model, for which the classical results are recalled in the appendix. Let us stress that the statement of Proposition 1 does not distinguish the number of unstable equilibriums nor the hyperbolic characteristics of the equilibriums, differently to [3]. Our goal here is simply to characterize the possible asymptotic behaviors towards a stable equilibrium, up to a set of initial conditions of null measure.

PROOF. Let us first note that one has $\dot{x}_{1}=0$ when $x_{1}=0$, and $\dot{x}_{2}=0$ when $x_{2}=0$. By uniqueness of the solutions of the system of differential equations 
(1), we deduce that the solutions verify $x_{1}(t)>0, x_{2}(t)>0$ for any $t>0$. At $s_{1}=0$, one has $\dot{s}_{1}=D s_{1}^{i n}>0$ and $\dot{s}_{2}>D s_{2}^{i n}>0$, which shows that the hyperplanes $s_{1}=0, s_{2}=0$ are repulsive. We deduce that the solutions verify $s_{1}(t)>0, s_{2}(t)>0$ for any $t>0$.

Consider the variables $z_{1}=x_{1}+s_{1}$ and $z_{2}=s_{1}+x_{2}+s_{2}$. From equations (1), one obtains

$$
\dot{z}_{1} \leq D\left(s_{1}^{i n}-\alpha z_{1}\right), \quad \dot{z}_{2} \leq D\left(s_{1}^{i n}+s_{2}^{i n}-\alpha z_{2}\right)
$$

from which one deduces that $z_{1}$ and $z_{2}$ are bounded. This shows that the non-negative solutions of (1) are bounded.

The $\left(x_{1}, s_{1}\right)$ dynamics is independent of the variables $x_{2}, s_{2}$ and follows the the classical chemostat model with monotonic growth function, whose steady state analysis is recalled in the Appendix (Proposition 4). Two cases are distinguished:

- case A: $\alpha D \geq \mu_{1}\left(s_{1}^{\text {in }}\right)$ : any solution of the $\left(x_{1}, s_{1}\right)$ sub-system converges to the wash-out state $E_{1}^{0}:=\left(0, s_{1}^{i n}\right)$.

- case B: $\alpha D<\mu_{1}\left(s_{1}^{\text {in }}\right)$ : any solution of the $\left(x_{1}, s_{1}\right)$ sub-system with $x_{1}(0)>0$ converges to the positive state $E_{1}^{\star}:=\left(\left(s_{1}^{i n}-\lambda_{1}(D)\right) / \alpha, \lambda_{1}(D)\right)$.

Then, the cascade structure of the dynamics (1), along with the boundedness of its solutions and the asymptotic behavior of the $\left(x_{1}, s_{1}\right)$ sub-system allows to proceed with the stability analysis of the system on the reduced dynamics of the $\left(x_{2}, s_{2}\right)$ subsystem:

$$
\left\{\begin{array}{l}
\dot{x}_{2}=\mu_{2}\left(s_{2}\right) x_{2}-\alpha D x_{2} \\
\dot{s}_{2}=-\mu_{2}\left(s_{2}\right) x_{2}+\mu_{1}\left(s_{1}^{e q}\right) x_{1}^{e q}+D\left(s_{2}^{i n}-s_{2}\right)
\end{array}\right.
$$

where $\left(x_{1}^{e q}, s_{1}^{e q}\right)$ is the steady state of the $\left(x_{1}, s_{1}\right)$ sub-system (i.e. $E_{1}^{0}$ or $E_{1}^{\star}$ when it exists, according to Proposition 4).

In case A, the subsystem (5) is the classical chemostat model with nonmonotonic growth, whose steady state analysis is recalled in Proposition 5 (see Appendix). Three cases are then possible depending on the value of $\alpha D$ with respect to $\mu_{2}^{m}$ and $\mu_{2}\left(s_{2}^{i n}\right)$, which are exactly the cases (1), (2), (3) given of the Proposition statement.

In case $\mathrm{B}$, notice that one has $\mu_{1}\left(s_{1}^{e q}\right) x_{1}^{e q}=D\left(s_{1}^{i n}-\lambda_{1}(D)\right)$ which allows to rewrite the reduced $\left(x_{2}, s_{2}\right)$ dynamics as

$$
\left\{\begin{array}{l}
\dot{x}_{2}=\mu_{2}\left(s_{2}\right) x_{2}-\alpha D x_{2} \\
\dot{s}_{2}=-\mu_{2}\left(s_{2}\right) x_{2}+D\left(\overline{s_{2}^{i n}}(D)-s_{2}\right)
\end{array}\right.
$$


where $\overline{s_{2}^{i n}}(D)$ is defined in (3). This is again the classical chemostat model but with non-monotonic growth and the effective input concentration $\overline{s_{2}^{i n}}(D)$, for which the steady state analysis given in Proposition 5 of the Appendix applies. This gives straightforwardly the cases (4), (5), (6) of the statement.

In practice, only case (6) is desirable because it guarantees that in any situation the wash-out of both species is avoided. Usually, the removal rate $D$ is the operating parameter that can be easily manipulated. In the next section, we study how to change the value of $D$ to be in case (6) when the original operating conditions are not in this case.

\section{Wash-out avoidance}

In this section, we consider situations for which the attraction basin of equilibria with wash-out of biomass 1 or 2 or both is non empty. According to Proposition 1, this happens in cases (1) to (5). We study now how to play only with the value of the removal rate $D$ to move to case (6).

Consider the domains

$\mathcal{D}_{5}:=\left\{\left(s_{1}^{i n}, s_{2}^{i n}, D\right) \in \mathbb{R}_{+}^{3} ; \alpha D<\mu_{1}\left(s_{1}^{i n}\right), \alpha D \leq \mu_{2}^{m}, \overline{s_{2}^{i n}}(D)>\lambda_{2}^{+}(D)\right\}$

$\mathcal{D}_{6}:=\left\{\left(s_{1}^{i n}, s_{2}^{i n}, D\right) \in \mathbb{R}_{+}^{3} ; \alpha D<\mu_{1}\left(s_{1}^{i n}\right), \alpha D \leq \mu_{2}^{m}, \lambda_{2}^{-}(D)<\overline{s_{2}^{i n}}(D) \leq \lambda_{2}^{+}(D)\right\}$

which are the sets of operating parameters $\left(s_{1}^{i n}, s_{2}^{i n}, D\right)$ that correspond to cases (5) and (6) of Proposition 1.

Consider the interval

$$
I:=\left(0, \min \left(\mu_{1}^{m}, \mu_{2}^{m}\right) / \alpha\right)
$$

and introduce the functions defined on $I$ :

$$
\nu^{-}(D):=\lambda_{1}(D)+\lambda_{2}^{-}(D), \nu^{+}(D):=\lambda_{1}(D)+\lambda_{2}^{+}(D), \quad D \in I
$$

(that are such that $\nu^{-}<\nu^{+}$on $I$ ), which allow to describe the domains $\mathcal{D}_{5}$, $\mathcal{D}_{6}$ as follows, using the expression $(3)$ of $\overline{s_{2}^{i n}}(D)$ when $\alpha D<\mu_{1}\left(s_{1}^{i n}\right)$.

$$
\begin{aligned}
& \mathcal{D}_{5}=\left\{\left(s_{1}^{i n}, s_{2}^{i n}, D\right) \in \mathbb{R}_{+}^{2} \times\left[0, \mu_{2}^{m} / \alpha\right] ; \alpha D<\mu_{1}\left(s_{1}^{i n}\right) ; \nu^{+}(D)<s_{1}^{i n}+s_{2}^{i n}\right\} \\
& \mathcal{D}_{6}=\left\{\left(s_{1}^{i n}, s_{2}^{i n}, D\right) \in \mathbb{R}_{+}^{2} \times\left[0, \mu_{2}^{m} / \alpha\right] ; \alpha D<\mu_{1}\left(s_{1}^{i n}\right) ; \nu^{-}(D)<s_{1}^{i n}+s_{2}^{i n} \leq \nu^{+}(D)\right\}
\end{aligned}
$$

Note from expressions (2) and (4) that the functions $\lambda_{1}$ and $\lambda_{2}^{-}$are increasing, while $\lambda_{2}^{+}$is decreasing. The function $\nu^{-}$is thus increasing. One has also 
$\nu^{-}(0)=0$ and $\nu^{-}(D) \rightarrow+\infty$ when $D \rightarrow \min \left(\mu_{1}^{m}, \mu_{2}^{m}\right) / \alpha$. One can then define the inverse function

$$
\eta^{-}(s):=\left(\nu^{-}\right)^{-1}(s), \quad s>0
$$

The function $\nu^{+}$is not necessarily monotonic but one has $\nu^{+}(D) \rightarrow+\infty$ when $D \rightarrow 0^{+}$. Denote

$$
s_{m}:=\min _{D \in \bar{I}} \nu^{+}(D)
$$

and one can define the function

$$
\eta^{+}(s):=\max \left\{D \in I ; \nu^{+}(D) \leq s\right\}, \quad s>s_{m} .
$$

Note that $\nu^{-}<\nu^{+}$on $I$ implies that one has $\eta^{-}(s)>\eta^{+}(s)$ for any $s>s_{m}$.

Our main result is the following.

Proposition 2 Consider a triplet $\left(s_{1}^{i n}, s_{2}^{i n}, D\right)$ with $s_{1}^{i n}>0, s_{2}^{i n} \geq 0$ and $D>0$, that do not belong to $\mathcal{D}_{6}$.

(i) For $\underline{D} \in(0, D)$ sufficiently small, $\left(s_{1}^{\text {in }}, s_{2}^{\text {in }}, \underline{D}\right)$ belongs to $\mathcal{D}_{6}$.

(ii) If $\left(s_{1}^{i n}, s_{2}^{i n}, D\right)$ belongs to $\mathcal{D}_{5}$ with the condition

$$
\alpha \eta^{+}\left(s_{1}^{i n}+s_{2}^{i n}\right)<\mu_{1}\left(s_{1}^{i n}\right)
$$

fulfilled, then any $\bar{D} \in\left(\eta^{+}\left(s_{1}^{i n}+s_{2}^{i n}\right), \min \left(\mu_{1}\left(s_{1}^{i n}\right) / \alpha, \eta^{-}\left(s_{1}^{i n}+s_{2}^{i n}\right)\right)\right.$ is such that $\bar{D}>D$ and $\left(s_{1}^{\text {in }}, s_{2}^{\text {in }}, \bar{D}\right)$ belongs to $\mathcal{D}_{6}$.

PROOF. Fix $s_{1}^{i n}>0$ and $s_{2}^{i n} \geq 0$.

Note first that $\nu^{-}(0)=0$ and $\nu^{+}(0+)=+\infty$ imply that one has $\nu^{-}(D)<$ $s_{1}^{i n}+s_{2}^{i n} \leq \nu^{+}(D)$ for $D>0$ small enough. Therefore, for any $D>0$, there exists $\underline{D} \in(0, D)$ such that $\left(s_{1}^{i n}, s_{2}^{i n}, d\right) \in \mathcal{D}_{6}$ for any $d \leq \underline{D}$. This shows (i).

Let us now study if it possible to have $\left(s_{1}^{i n}, s_{2}^{i n}, \bar{D}\right) \in \mathcal{D}_{6}$ with $\bar{D}>D$ when $D>0$ is such that $\left(s_{1}^{i n}, s_{2}^{i n}, D\right) \notin \mathcal{D}_{6}$.

According to Proposition 1, in cases (1), (2) or (3), one has $\alpha D \geq \mu_{1}\left(s_{1}^{i n}\right)$ and $D$ has then to be reduced to fulfill the condition $\alpha D<\mu_{1}\left(s_{1}^{\text {in }}\right)$ required in case (6).

In case (4), either one has $\alpha D>\mu_{2}^{m}$, and $D$ has to be reduced to fulfill the condition $\alpha D \leq \mu_{2}^{m}$ of case (6), or one has $\overline{s_{2}^{i n}}(D) \leq \lambda_{2}^{-}(D)$. This latter situation amounts to write $s_{1}^{i n}+s_{2}^{i n} \leq \nu^{-}(D)$ and as the function $\nu^{-}$is increasing, $D$ has again to be reduced to obtain the condition $s_{1}^{i n}+s_{2}^{i n}>\nu^{-}(D)$ of case (6). 
In case (5), one has $s_{1}^{i n}+s_{2}^{i n}>\nu^{+}(D)$ which implies $D<\eta^{+}\left(s_{1}^{i n}+s_{2}^{\text {in }}\right)$ by definition of $\eta^{+}$. If $\alpha \eta^{+}\left(s_{1}^{i n}+s_{2}^{i n}\right)<\mu_{1}\left(s_{1}^{i n}\right)$, then any $\bar{D}$ in the interval $\left(\eta^{+}\left(s_{1}^{i n}+s_{2}^{i n}\right), \mu_{1}\left(s_{1}^{i n}\right) / \alpha\right)$ verifies $s_{1}^{i n}+s_{2}^{i n} \leq \nu^{+}(\bar{D})$. If moreover one has $\bar{D}<$ $\eta^{-}\left(s_{1}^{i n}+s_{2}^{i n}\right)$ (recall that $\left.\eta^{+}<\eta^{-}\right)$, one guarantees the inequality $s_{1}^{i n}+s_{2}^{i n}<$ $\eta^{-}(\bar{D})$. This proves the point (ii).

The surprising fact, when compared to the usual chemostat model, is that increasing the removal rate $D$ can bring stability in certain situations given in (ii). Indeed, increasing the removal rate amounts to reduce the residence time, which is usually a factor of instability. Here, the key point for such a phenomenon to occur relies on the possible non monotonicity of the function $\nu^{+}$, which implies that the function $\eta^{+}$is non identically equal to $\min \left(\mu_{1}^{m}, \mu_{2}^{m}\right) / \alpha$. Let us show that the conditions of case (ii) of Proposition 2 can be really met.

Proposition 3 Assume one has $\mu_{2}^{m} \geq \mu_{1}^{m}$. Let

$$
D_{m}:=\max \left\{D \in I ; \nu^{+}(D)=s_{m}\right\} .
$$

(i) Any triplet $\left(s_{1}^{i n}, s_{2}^{i n}, D\right)$ such that $s_{1}^{i n}+s_{2}^{i n}>s_{m}$ and $D>D_{m}$ with $\nu^{+}(D)<s_{1}^{\text {in }}+s_{2}^{\text {in }}$ belongs to $\mathcal{D}_{5}$ and verifies the inequality (7), provided that $s_{2}^{\text {in }}$ is sufficiently small.

(ii) Any triplet $\left(s_{1}^{i n}, 0, D\right)$ that belongs to $\mathcal{D}_{5}$ satisfies the inequality (7).

\section{PROOF.}

Notice first that when $\mu_{2}^{m} \geq \mu_{1}^{m}, \mu_{1}^{m}$ is necessarily finite and one has $I=$ $\left(0, \mu_{1}^{m} / \alpha\right)$.

Take any $s>s_{m}$ and denote $\tilde{D}=\eta^{+}(s)$. For any $D>D_{m}$ in $I$ such that $\nu^{+}(D)<s$, one has

$$
\lambda_{1}(D)<s-\lambda_{2}^{+}(D) .
$$

As $D<\tilde{D}$ and $\lambda_{2}^{+}$is decreasing, one has also

$$
\lambda_{1}(D)<s-\lambda_{2}^{+}(\tilde{D}) .
$$

Consider now any $s_{2}^{i n}<\min \left(s, \lambda_{2}^{+}(\tilde{D})\right)$ and take $s_{1}^{i n}=s-s_{2}^{i n}$. For this choice of $s_{1}^{i n}$ and $s_{2}^{\text {in }}$, inequality (8) gives

$$
\lambda_{1}(D)<s_{1}^{i n}
$$

(along with $\left.\nu^{+}(D)<s_{1}^{i n}+s_{2}^{i n}\right)$. As the interval $I$ is equal to $\left(0, \mu_{1}^{m} / \alpha\right)$ and the function $\mu_{1}$ is increasing, having $\lambda_{1}(D)<s_{1}^{\text {in }}$ is equivalent to have $\alpha D<$ 
$\mu_{1}\left(s_{1}^{i n}\right)$. This shows that the triplet $\left(s_{1}^{i n}, s_{2}^{i n}, D\right)$ belongs to $\mathcal{D}_{5}$ (notice that one has necessarily $\alpha D<\mu_{2}^{m}$ as $\left.\mu_{1}^{m} \leq \mu_{2}^{m}\right)$.

For $D=\tilde{D}$, one has $\nu^{+}(\tilde{D})=s$. In the same manner, one obtains $\lambda_{1}(\tilde{D})<s_{1}^{\text {in }}$ for the former choice of $s_{1}^{i n}$ and $s_{2}^{i n}$, or equivalently $\alpha \tilde{D}<\mu_{1}\left(s_{1}^{i n}\right)$. This shows that the inequality

$$
\alpha \eta^{+}\left(s_{1}^{i n}+s_{2}^{i n}\right)<\mu_{1}\left(s_{1}^{i n}\right)
$$

is necessarily fulfilled.

For the particular case $s_{2}^{i n}=0$, note that $\nu^{+}(D)<s_{1}^{i n}$ implies $\lambda_{1}(D)<s_{1}^{\text {in }}$ which, in turns, implies $\alpha D<\mu_{1}\left(s_{1}^{i n}\right)$. From the definition of $\nu^{+}$, this shows that the inequality $\alpha \eta^{+}\left(s_{1}^{i n}\right)<\mu_{1}\left(s_{1}^{i n}\right)$ is verified.

\section{Numerical illustrations}

Typical instances of functions that fulfill Assumption 1 are given by the Monod expression for the first reaction

$$
\mu_{1}\left(s_{1}\right)=\frac{\mu_{1}^{m} s_{1}}{K_{1}+s_{1}} .
$$

and the Haldane one for the second

$$
\mu_{2}\left(s_{2}\right)=\frac{\bar{\mu}_{2} s_{2}}{K_{2}+s_{2}+\frac{s_{2}^{2}}{K_{i}}}
$$

for which one has

$$
\hat{s}_{2}=\sqrt{K_{2} K_{i}}
$$

Then, the break even concentrations defined in Section 2 have the expressions

$$
\lambda_{1}(D)=\frac{\alpha D K_{1}}{\mu_{1}^{m}-\alpha D}, \quad \alpha D<\mu_{1}^{m}
$$

for the Monod function (9), and for the Haldane function (10)

$$
\lambda_{2}^{ \pm}(D)=\frac{\bar{\mu}_{2}-\alpha D \pm \sqrt{\left(\bar{\mu}_{2}-\alpha D\right)^{2}-4(\alpha D)^{2} \frac{K_{2}}{K_{i}}}}{2 \frac{\alpha D}{K_{i}}}, \quad \alpha D \leq \mu_{2}^{m}
$$

Figure 1 gives the values of the parameters chosen for the numerical computation, along with the graphs of the corresponding functions $\mu_{1}, \mu_{2}$. The numerical values of the parameters were chosen such that the graphical representations be very clear and the phenomenon very well put in evidence. We 
will come back on this point in the discussion at the end of this section but the phenomenon appears for several parameter sets reported in the literature even if it may be less obvious and more difficult to represent graphically (cf. for instance [13]) as well as for more complicated models, (cf. [7, 19] for example).

\begin{tabular}{c|c||c|c|c||c}
$\mu_{1}^{m}$ & $K_{1}$ & $\bar{\mu}_{2}$ & $K_{2}$ & $K_{i}$ & $\alpha$ \\
\hline 0.6 & 0.4 & 1.1 & 1 & 7 & 0.7
\end{tabular}

(a) Parameters values.

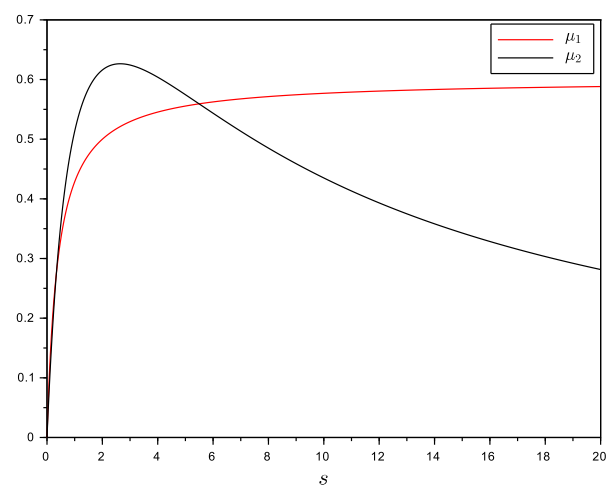

(b) Graphs of $\mu_{1}$ and $\mu_{2}$.

Fig. 1.

For these values, one has $\hat{s}_{2} \simeq 2.646$ and $\mu_{2}^{m} \simeq 0.626$. So, we are in the case $\mu_{2}^{m}>\mu_{1}^{m}$ of Proposition 3 .

Figure 2 gives the graphs of the associated break-even concentrations $\lambda_{1}, \lambda_{2}^{ \pm}$ and the functions $\nu^{ \pm}$.
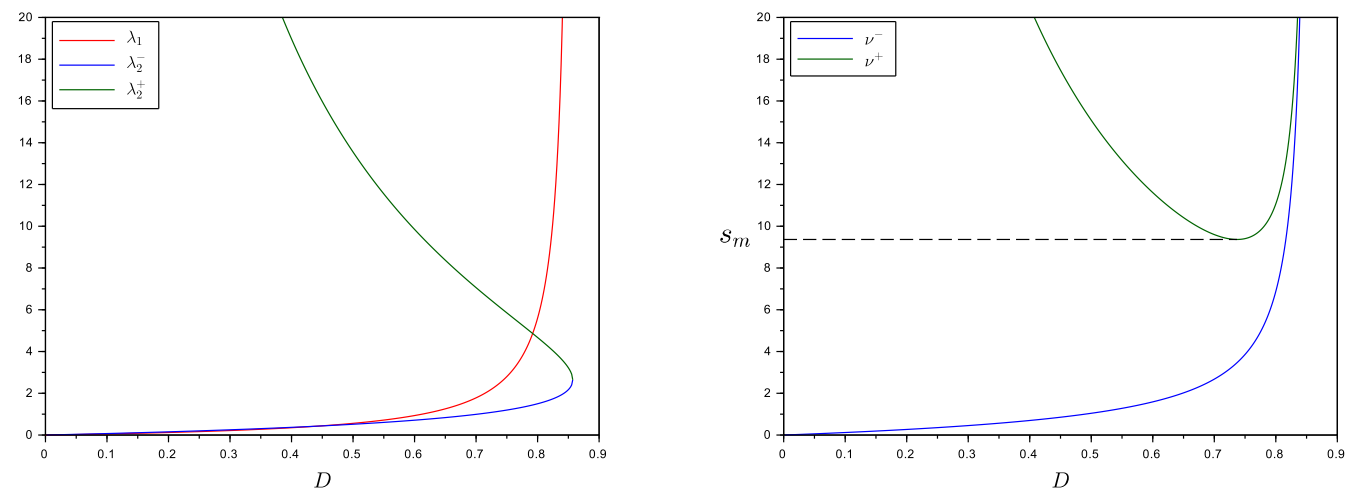

Fig. 2. Graphs of the functions $\lambda_{i}^{ \pm}$(left) and $\nu^{ \pm}=\lambda_{1}+\lambda_{2}^{ \pm}$(right).

Remind that $\lambda_{1}$ and $\lambda_{2}^{-}$are increasing while $\lambda_{2}^{+}$is decreasing. The function $\nu^{+}$ is always above $\nu^{-}$(which is increasing) and $\nu^{+}$tends to $+\infty$ on the boundary of the interval $I=\left(0, \mu_{1}^{m} / \alpha\right)$ when $\mu_{1}^{m} \leq \mu_{2}^{m}$.

As it is not always easy to grasp domains in $\mathbb{R}^{3}$, we fix values of $s_{2}^{\text {in }}$ and depict 
the cross-sections of the domains $\mathcal{D}_{5}, \mathcal{D}_{6}$ in the $\left(s_{1}^{i n}, D\right)$ plane. For a fixed value of $s_{2}^{i n}$, these cross-sections are

$$
\begin{aligned}
& \mathcal{C}_{5}\left(s_{2}^{i n}\right):=\left\{\left(s_{1}^{i n}, D\right) \in \mathbb{R}_{+} \times I, \alpha D<\mu_{1}\left(s_{1}^{i n}\right) ; \nu^{+}(D)<s_{1}^{i n}+s_{2}^{i n}\right\} \\
& \mathcal{C}_{6}\left(s_{2}^{i n}\right):=\left\{\left(\left(s_{1}^{i n}, D\right) \in \mathbb{R}_{+} \times I, \alpha D<\mu_{1}\left(s_{1}\right)^{i n}\right) ; \nu^{-}(D)<s_{1}^{i n}+s_{2}^{i n} \leq \nu^{+}(D)\right\}
\end{aligned}
$$

It can be simply interpreted in terms of intersections of epigraphs or hypographs of the functions $\lambda_{1}, \nu^{+}-s_{2}^{i n}$ and $\nu^{-}-s_{2}^{i n}$ in the $\left(D, s_{1}^{i n}\right)$ symmetric plane:

$$
\begin{aligned}
& \mathcal{C}_{5}\left(s_{2}^{i n}\right):=\left\{s_{1}^{i n}>\lambda_{1}(D)\right\} \cup\left\{s_{1}^{i n}>\nu^{+}(D)-s_{2}^{i n}\right\} \\
& \mathcal{C}_{6}\left(s_{2}^{i n}\right):=\left\{s_{1}^{i n}>\lambda_{1}(D)\right\} \cup\left\{s_{1}^{i n} \leq \nu^{+}(D)-s_{2}^{i n}\right\} \cup\left\{s_{1}^{i n}>\nu^{-}(D)-s_{2}^{i n}\right\}
\end{aligned}
$$

Accordingly to Proposition 2, the complementary of $\mathcal{C}_{5}\left(s_{2}^{i n}\right) \cup \mathcal{C}_{6}\left(s_{2}^{i n}\right)$ in the domain $\left\{\left(s_{1}^{i n}, D\right) \in \mathbb{R}_{+} \times I ; \alpha D<\mu_{1}\left(s_{1}^{i n}\right)\right\}$ corresponds to case (4) and the complementary $\left\{\left(s_{1}^{i n}, D\right) \in \mathbb{R}_{+}^{2} ; \alpha D \geq \mu_{1}\left(s_{1}^{i n}\right) s\right\}$ is covered by cases (1), (2) and (3).

In Figure 3, one can see that for $s_{2}^{\text {in }}=0, \mathcal{C}_{5}$ and $\mathcal{C}_{6}$ cover almost but not all the domain $\left\{\alpha D<\mu_{1}\left(s_{1}^{i n}\right)\right\}$ (which is below the red curve), and that it is always possible to reach the domain $\mathcal{C}_{6}$ (in green) from $\mathcal{C}_{5}$ (in pink) by increasing $D$ (for fixed $s_{1}^{i n}$ ), in accordance with Proposition 3 . The red curve, which is the graph of the function $\mu_{1} / \alpha$, is above the domain $\mathcal{C}_{5}$.

For $s_{2}^{i n}=2$ or $s_{2}^{i n}=5, \mathcal{C}_{5}$ and $\mathcal{C}_{6}$ cover all the domain $\left\{D<\mu_{1}\left(s_{1}^{i n}\right) / \alpha\right\}$ and the graph of the red curve touches the boundary $\mathcal{C}_{5}$ (see Figure 4). However, it still possible to reach $\mathcal{C}_{6}$ from $\mathcal{C}_{5}$ by increasing $D$ only if $s_{1}^{\text {in }}$ is close enough to the value $s_{m}-s_{2}^{i n}$ (remind that $s_{m}$ is the minimum of the function $\nu^{+}$).

Increasing the values of $s_{2}^{\text {in }}$ slides further the cross-section $\mathcal{C}_{5}$ to the left on the $\left(s_{1}^{\text {in }}, D\right)$ plane and it is no longer possible to reach $\mathcal{C}_{6}$ from $\mathcal{C}_{5}$ by simply increasing $D$ (see Figure 5), exactly when the extreme point $\left(s_{m}-s_{2}^{i n}, D_{m}\right)$ of $\mathcal{C}_{5}$ does no longer lie in the domain $\left\{\alpha D<\mu_{1}\left(s_{1}^{i n}\right)\right\}$ (i.e. when it is no longer located below the red curve). Then, the only way to stabilize the system playing only with the removal rate $D$ is to decrease its value such that $\left(s_{1}^{\text {in }}, D\right)$ is below the green curve (which is the graph of the function $\nu^{+}-s_{2}^{i n}$ ).

Notice that this interesting property of two-step processes was already visible in certain figures of some papers of the literature, notably in relation to the analysis of the anaerobic digestion process (cf. for instance the Figure 4 of the paper by Sbarciog et al., 2010, [13]). However, the property was not underlined in the paper as a way to stabilize the system. It should be noticed that this property obviously appears in more complex models. In [7], who studied a 


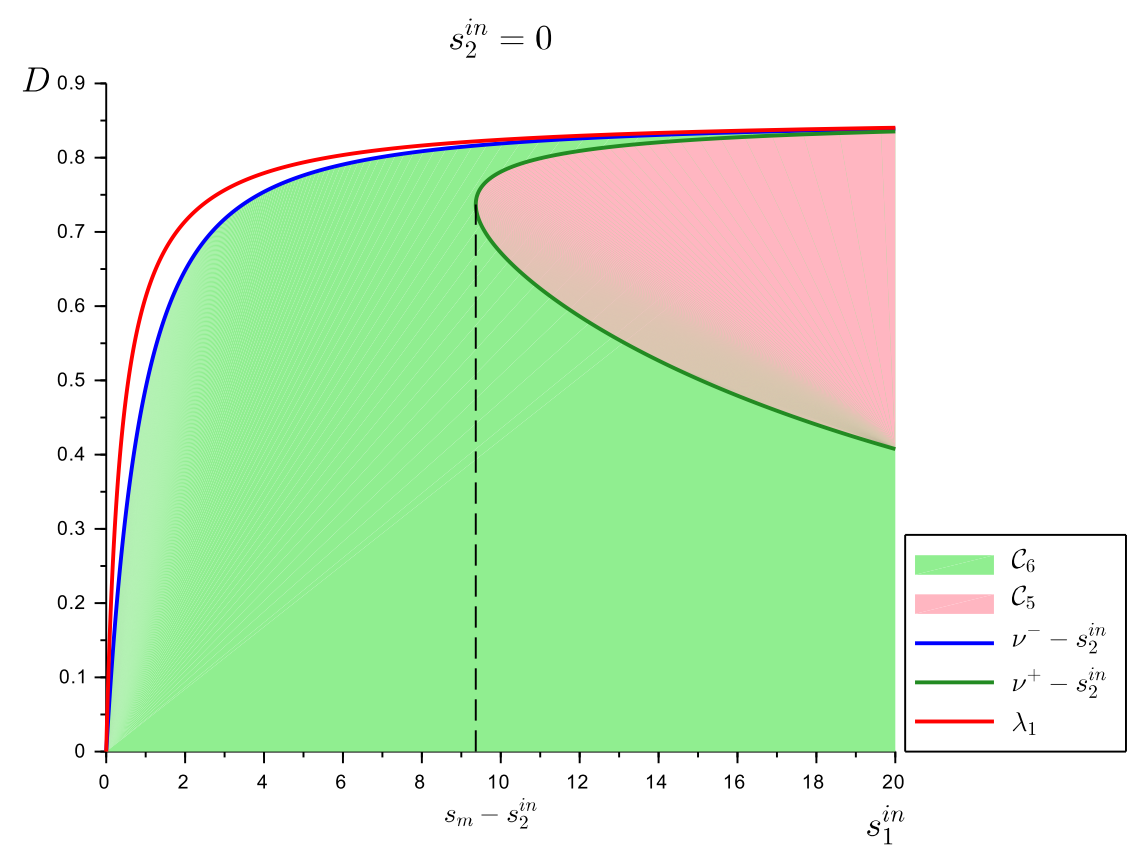

Fig. 3. Cross-sections of the domains $\mathcal{D}_{5}$ and $\mathcal{D}_{6}$ for $s_{2}^{i n}=0$. From any operating point $\left(s_{1}^{i n}, D\right) \in \mathcal{C}_{5}$ it is possible to reach $\mathcal{C}_{6}$ by simply increasing $D$.
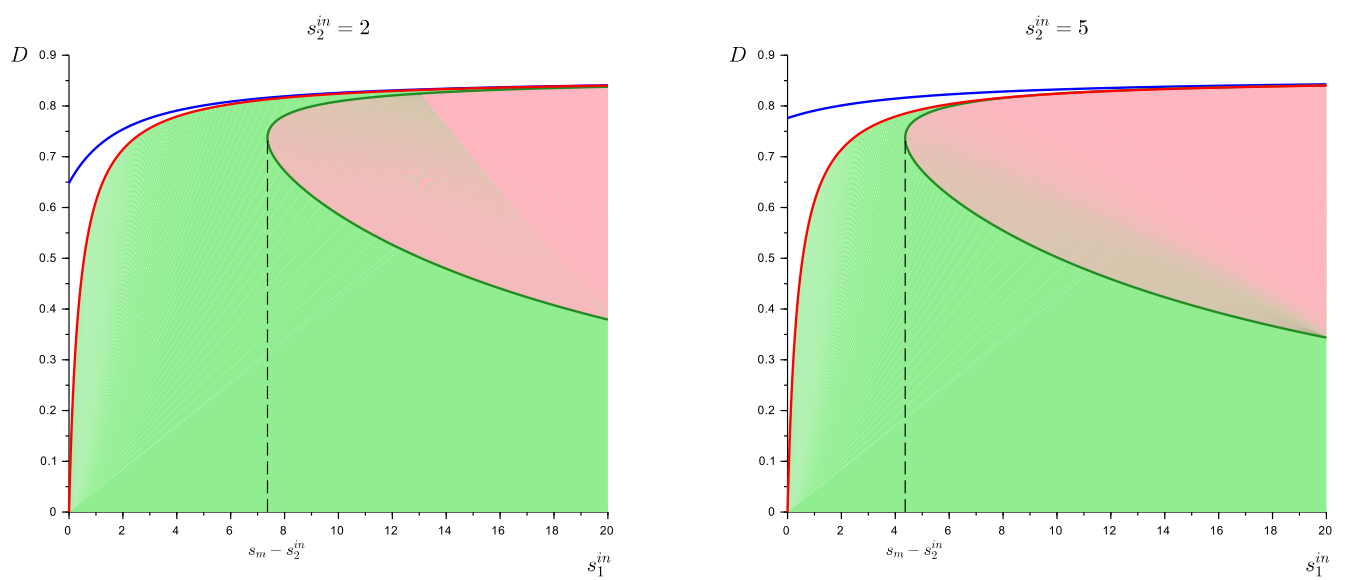

Fig. 4. Cross-sections of the domains $\mathcal{D}_{5}$ and $\mathcal{D}_{6}$ for $s_{2}^{\text {in }}=2$ and $s_{2}^{i n}=5$. From an operating point $\left(s_{1}^{\text {in }}, D\right) \in \mathcal{C}_{5}$ with $s_{1}^{\text {in }}$ not too far from $s_{m}-s_{2}^{\text {in }}$, it is possible to reach $\mathcal{C}_{6}$ by simply increasing $D$.

model of the anaerobic digestion of microalgae, this property was highlighted. In particular, when comparing the two models of the anaerobic digestion initially proposed in [4] and [5], it was first noticed that the inhibition coefficient identified in the first model, ([4]) was too large to play a role in the system dynamics (recall that having a large inhibition coefficient in the Haldane function is equivalent to considering a Monod kinetics). Then, in modifying this coefficient (increasing the inhibition of the second step kinetics in decreasing 
the value of the inhibition coefficient in the Haldane kinetics), it was possible to show that the region in which the biogas is maximum exhibits a bistability. Comparing these results with those obtained when studying the second model (from [5]) yielded to plot the Figure 11 of [7] where this property is visible (but not further discussed). Finally, this property has been described in [12] for two-step biological systems with density-dependent kinetics in the first step, but again without giving any condition to test it nor proposing a control strategy to take advantage of it. Here we give sufficient conditions that can easily be tested for the phenomenon to appear.

Notice that the proposed strategy consists in decreasing the residence time and not increasing it as usually done to stabilize bio-processes. This has some practical advantages: it allows to treat a larger quantity of matter and for industrial plants which impose a minimal flow rate to be processed, it does not require any upstream storage. Of course, increasing the residence time increases the output concentration at steady-state, but once about the steadystate one may reduce the flow rate to its nominal value.
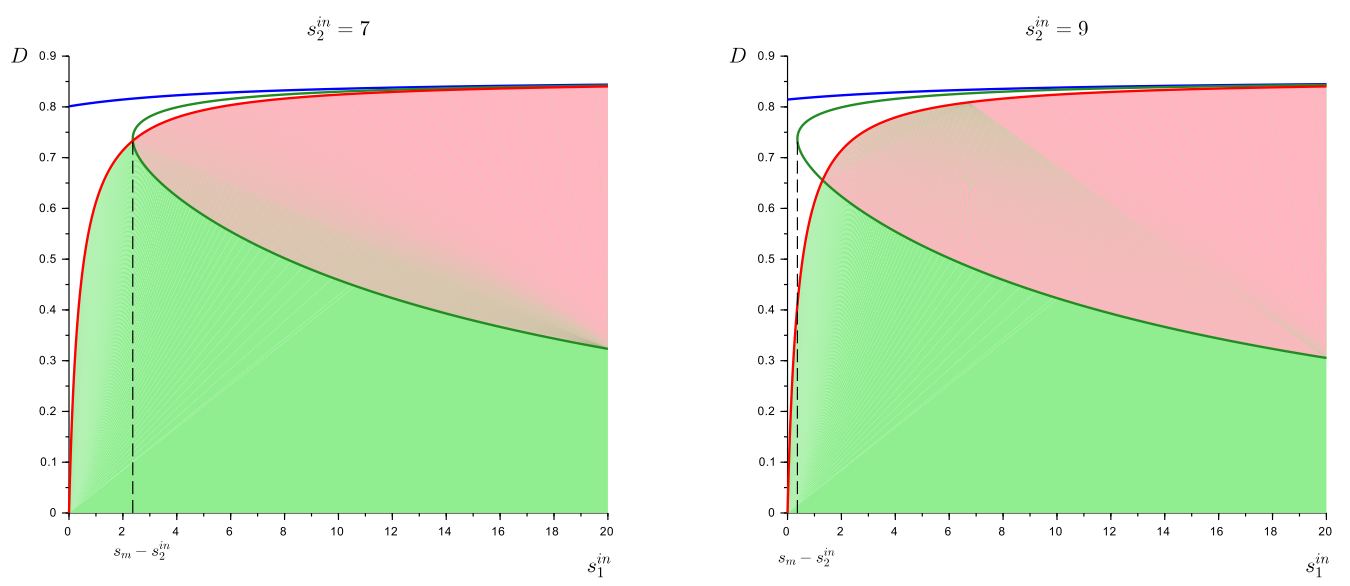

Fig. 5. Cross-sections of the domains $\mathcal{D}_{5}$ and $\mathcal{D}_{6}$ for $s_{2}^{i n}=7$ and $s_{2}^{i n}=9$. From any operating point $\left(s_{1}^{i n}, D\right) \in \mathcal{C}_{5}$ it it not possible to reach $\mathcal{C}_{6}$ by simply increasing $D$.

\section{Conclusion}

This study reveals the role played by the sum of the break-even concentrations, as the function $D \mapsto \lambda_{1}(D)+\lambda_{2}^{+}(D)$, in the counter-intuitive phenomenon of increasing the removal rate to stabilize a two-steps bioprocess model. More precisely, we show that when this function is non-monotonic on its domain, this phenomenon occurs provided that the input concentration $s_{2}^{i n}$ of substrate of the second reaction is null of not too large. This result provides a new way to stabilize such processes in certain situations, without increasing the residence time, as it is often done which may be penalizing in an industrial context. 


\section{Appendix}

We recall here the classical results about the chemostat model

$$
\left\{\begin{array}{l}
\dot{x}=\mu(s) x-\alpha D x \\
\dot{s}=-\mu(s) x+D\left(s^{i n}-s\right)
\end{array}\right.
$$

when $\mu$ is a monotonic or non-monotonic function.

Proposition 4 Assume that the function $\mu$ is increasing on $\left(0, s^{\text {in }}\right)$ and define the break-even concentration $\lambda(D)$ such that

$$
\mu(\lambda(D))=\alpha D, \quad \alpha D \in\left(0, \mu\left(s^{i n}\right)\right)
$$

- When $\alpha D \geq \mu\left(s^{i n}\right)$, the system (11) has an unique equilibrium $E^{0}:=\left(0, s^{i n}\right)$ ("wash-out"), which is moreover globally asymptotically stable on $\mathbb{R}_{+}^{2}$.

- When $\alpha D<\mu\left(s^{i n}\right)$, the system (11) admits an unique positive equilibrium $E^{+}:=\left(\left(s^{i n}-\lambda(D)\right) / \alpha, \lambda(D)\right)$ (in addition to the equilibrium $\left.E^{0}\right)$, which is moreover globally asymptotically stable on the domain $\mathbb{R}_{+}^{*} \times \mathbb{R}_{+}$.

Proposition 5 Assume that there exists $\hat{s} \in\left(0, s^{i n}\right)$ such that the function $\mu$ is increasing on $(0, \hat{s})$ and decreasing on $\left(\hat{s}, s^{i n}\right)$. Define the break-even concentrations $\lambda^{-}(D), \lambda^{+}(D)$ as follows

$$
\begin{aligned}
& \lambda^{-}(D)=\min \{s \in[0, \hat{s}] ; \mu(s) \geq \alpha D\}, \quad \alpha D \in[0, \mu(\hat{s})] \\
& \lambda^{+}(D)=\max \left\{s \in\left[\hat{s}, s^{i n}\right] ; \mu(s) \geq \alpha D\right\}, \quad \alpha D \in\left[\mu\left(s^{i n}\right), \mu(\hat{s})\right]
\end{aligned}
$$

- If $\alpha D>\mu(\hat{s})$, the system (11) has the unique equilibrium $E^{0}:=\left(0, s^{i n}\right)$, which is globally asymptotically stable on $\mathbb{R}_{+}^{2}$.

- If $\alpha D<\mu\left(s^{i n}\right)$, the system (11) admits an unique positive equilibrium $E^{-}:=$ $\left(\left(s^{i n}-\lambda^{-}(D)\right) / \alpha, \lambda^{-}(D)\right)$ which is globally asymptotically stable on $\mathbb{R}_{+}^{\star} \times \mathbb{R}_{+}$.

- If $\alpha D \in\left[\mu\left(s^{i n}\right), \mu\left(s^{\star}\right)\right]$, the system (11) presents a bi-stability between $E^{-}$ and $E^{0}$. From any initial condition in $\mathbb{R}_{+}^{\star} \times \mathbb{R}_{+}$excepted on a set of null measure, the solution converges asymptotically to $E^{-}$or $E^{0}$.

\section{Acknowledgments}

The work has been achieved in the framework of the PHC program 'TOURNESOL' 2018-19 between France and Belgium-Wallonia. The authors are very grateful to Professor Tewfik Sari for fruitful discussions. 


\section{References}

[1] G. Bastin, D. Dochain (1990) On-line Estimation and Adaptive Control of Bioreactors, Elsevier.

[2] D.J. Batstone, J. Keller, I. Angelidaki, S.V. Kalyzhnyi, S.G. Pavlostathis, A.Rozzi, W.T.M. Sanders, H. Siegrist, V.A. Vavilin (2002) The Iwa Anaerobic Digestion Model No 1 (ADM1). Water Science and Technology 45, 65-73.

[3] B. Benyahia, T. Sari, B. Cherki, J. Harmand (2012) Bifurcation and stability analysis of a two step model for monitoring anaerobic digestion processes. J. Process Control 22, 1008-1019.

[4] O. Bernard, Z. Hadj-Sadock, D. Dochain, A. Genovesi, J.-P. Steyer (2001) Dynamical model development and parameter identification for an anaerobic wastewater treatment process. Biotechnology and Bioengineering 75, 424-438.

[5] F. Mairet, O. Bernard, E. Cameron, M. Ras, L. Lardon, J.P. Steyer, B. Chachuat (2011) Three-reaction model for the anaerobic digestion of microalgae, J. Biotechnol. Bioeng. 109, 415-429.

[6] J. Harmand, C. Lobry, A. Rapaport, T. Sari (2017) The Chemostat: Mathematical Theory of Microorganism Cultures, John Wiley \& Sons.

[7] Z. Khedim, B. Benyahia, B. Cherki, T. Sari, J. Harmand (2018) Effect of control parameters on biogas production during the anaerobic digestion of protein-rich substrates, Applied Mathematical Modelling 61, 351-376.

[8] A. Rapaport, J. Harmand (2002) Robust regulation of a class of partially observed nonlinear continuous bioreactors. J. Process Control 12, 291-302.

[9] A. Schaum, J. Alvarez, T. Lopez-Arenas (2012) Saturated PI control of continuous bioreactors with Haldane kinetics, Chemical Engineering Science 68(1), 520-529.

[10] I. Ramirez, A. Mottet, H. Carrère, S. Déléris, F. Vedrenne, J.P. Steyer (2009) Modified ADM1 disintegration/hydrolysis structures for modeling batch thermophilic anaerobic digestion of thermally pretreated waste activated sludge, Water Research, 43 (14), 3479-3492.

[11] P.J. Reilly (1974) Stability of commensalistic systems, Biotechnology and Bioengineering 16, 1373-1392.

[12] M. Hanaki, J. Harmand, Z. Mghazli, T. Sari, A. Rapaport, P. Ugalde, Mathematical study of a two-stage anaerobic model when the hydrolysis is the limiting step, preprint hal .archives-ouvertes.fr/hal-02531141.

[13] M. Sbarciog, M. Loccufier, E. Noldus (2010) Determination of appropriate operating strategies for anaerobic digestion systems, Biochemical Engineering Journal 51 (3), 180-188.

[14] T. Sari, B. Benyahia (2020) The operating diagram for a two-step anaerobic digestion model, preprint hal. archives-ouvertes.fr/hal-02557464.

[15] T. Sari, J. Harmand (2016) A model of a syntrophic relationship between two microbial species in a chemostat including maintenance, Mathematical 
Biosciences 275, 1-9.

[16] B. Satishkumar, M. Chidambaram (1999) Control of unstable bioreactor using fuzzy-tuned PI controller, Bioproc. Eng. 20, 127-132.

[17] G. Stephanopoulos (1981) The dynamic of commensalism, Biotechnology and Bioengineering 23, 2243-2255.

[18] V.A. Vavilin, B. Fernandez, J.Palatsi, X.Flotats (2008) Hydrolysis kinetics in anaerobic degradation of particulate organic material: An overview, Waste Management 28, 939-951.

[19] E. Volcke, M. Sbarciog, E.J.L. Noldus, B. De Baets, M. Loccufier (2010) Steady-state multiplicity of two-step biological conversion systems with general kinetics, Mathematical Biosciences 228, 160-170.

[20] M.J. Wade, J. Harmand, B. Benyahia, T. Bouchez, S. Chailou, B. Cloez, J-J. Godon, B. Moussa Boudjmaa, A. Rapaport, T. Sari, R. Arditi, C. Lobry (2016). Perspectives in mathematical modelling for microbial ecology, Ecological Modelling 321, 64-74.

[21] M. Weedermann, G. Seo, G. Wolkowics (2013) Mathematical Model of Anaerobic Digestion in a Chemostat: Effects of Syntrophy and Inhibition, Journal of Biological Dynamics 7, 59-85.

[22] M. Weedermann, G. Wolkowicz, J. Sasara (2015) Optimal biogas production in a model for anaerobic digestion, Nonlinear Dynamics 81, 1097-1112. 